\title{
Relationship between Dementia Severity and Behavioral and Psychological Symptoms of Dementia in Dementia with Lewy Bodies and Alzheimer's Disease Patients
}

\author{
Mamoru Hashimoto ${ }^{a}$ Yusuke Yatabe $^{a}$ Tomohisa Ishikawa ${ }^{a}$ Ryuji Fukuhara ${ }^{a}$ b \\ Keiichiro Kaneda ${ }^{a}$ Kazuki Honda ${ }^{a}$ Seiji Yuki ${ }^{a}$ Yusuke Ogawa ${ }^{a}$ \\ Toru Imamurac Hiroaki Kazui ${ }^{c}$ Naoto Kamimura ${ }^{\mathrm{e}}$ Syunichiro Shinagawa ${ }^{f}$ \\ Katsuyoshi Mizukami ${ }^{9}$ Etsuro Mori ${ }^{\mathrm{h}}$ Manabu Ikeda ${ }^{\mathrm{a}}$

\begin{abstract}
a Department of Neuropsychiatry, Faculty of Life Sciences, Kumamoto University, Kumamoto, ${ }^{b}$ Department of Neuropsychiatry, Ehime University Graduate School of Medicine, Toon, 'Division of Speech, Hearing and Cognitive Sciences, Graduate School of Health and Welfare, Niigata University of Health and Welfare, Niigata, 'Psychiatry, Department of Integrated Medicine, Division of Internal Medicine, Osaka University, Suita, e Department of Neuropsychiatry, Kochi Medical School, Nankoku, ${ }^{f}$ Department of Psychiatry, Jikei University School of Medicine, and ${ }^{9}$ Graduate School of Comprehensive Human Sciences, University of Tsukuba, Tokyo, and ${ }^{h}$ Department of Behavioral Neurology and Cognitive Neuroscience, Tohoku University Graduate School of Medicine, Sendai, Japan
\end{abstract}

\section{Key Words}

Dementia with Lewy bodies · Alzheimer's disease · Neuropsychiatric symptoms · Severity of dementia

\section{Abstract}

Background/Aims: Behavioral and psychological symptoms of dementia (BPSD) are common in the clinical manifestation of dementia. Although most patients with dementia exhibit some BPSD during the course of the illness, the association of BPSD with the stage of dementia remains unclear. It was the aim of this study to evaluate the impact of severity of dementia on the expression of BPSD in patients with dementia with Lewy bodies (DLB) and Alzheimer's disease (AD). Methods: Ninety-seven patients with DLB and 393 patients with AD were recruited from 8 dementia clinics across Japan. BPSD were assessed by the Neuropsychiatric Inventory (NPI). A relationship between BPSD and dementia stage classified by the Clinical Dementia Rating (CDR) in each type of dementia was assessed. Results: No significant difference was seen in NPI total score across CDR staging in the DLB group. On the other hand, the 
NPI total score significantly increased with dementia stage in the AD group. Conclusion: The relationship of dementia stage with the expression of BPSD was different according to the type of dementia. BPSD and dementia stage were correlated in AD subjects, in whom psychiatric symptoms increase as the disease progresses, but not in DLB subjects.

(C) 2015 S. Karger AG, Basel

\section{Introduction}

Behavioral and psychological symptoms of dementia (BPSD) is a descriptive term that embraces a heterogeneous group of noncognitive symptoms and behaviors occurring in patients with dementia [1]. BPSD includes delusions and hallucinations, disinhibited behavior, verbal and physical aggression, anxiety and depression, sleep disturbance, and other manifestations [2]. The development of BPSD is associated with more rapid cognitive decline [3], greater impairment in activities of daily living [4], lower quality of life for patients and caregivers [5], and earlier institutionalization [6].

Several studies have reported that different types of dementia present with characteristic behavioral profiles that reflect the specific brain regions affected. Furthermore, recent studies have found that the features of BPSD might be influenced by dementia stage [7-10]. For example, in patients with Alzheimer's disease (AD), mood symptoms generally develop early in the course of the disease; on the other hand, psychotic symptoms and agitation characterize the middle to late stages of dementia [8]. Most patients with dementia exhibit some BPSD at some time during the course of the illness. Nevertheless, the relationship between BPSD and the stage of dementia remains unclear.

Dementia with Lewy bodies (DLB) is the second most common form of neurodegenerative dementia after $\mathrm{AD}[11,12]$. It is well known that psychotic symptoms such as visual hallucinations and a variety of delusions occur more frequently in DLB than in AD from the early disease stage $[13,14]$. However, the longitudinal course of these symptoms has received little concern, and the results have been inconsistent. Some clinical studies have demonstrated that BPSD are closely related to the severity of dementia in DLB $[15,16]$. On the other hand, a neuropathological study reported that BPSD were equally common in different stages of DLB [13]. In the present study, we investigated the relationship between dementia stage and BPSD in patients with DLB and AD.

\section{Methods}

\section{Subjects}

All procedures followed the Clinical Study Guidelines of the Ethics Committee of Kumamoto University and were approved by the Internal Review Board. After a complete description of all procedures of the present study, written informed consent was obtained from patients or their caregivers.

This study used a cross-sectional design. The study population consisted of 97 patients with DLB and 393 patients with AD who attended 8 dementia outpatient clinics across Japan between January 2010 and October 2011. Patients underwent general physical, neurological, and neuropsychological examinations including the Mini-Mental State Examination (MMSE) [17]. All underwent structural neuroimaging with magnetic resonance imaging or computed tomography of the brain and routine laboratory testing including vitamin $\mathrm{B}_{1}$, vitamin $\mathrm{B}_{12}$, and thyroid function. Clinical diagnoses were made by experienced senior neuropsychiatrists or neurologists according to the following consensus criteria. 
Hashimoto et al.: Relationship between Dementia Severity and Behavioral and

The diagnosis of dementia was made according to the criteria of the Diagnostic and Statistical Manual of Mental Disorders, 3rd edition-revised (DSM-III-R) [18]. Probable DLB was diagnosed on the basis of the Consortium on DLB International Workshop criteria [19]. Patients were diagnosed as having AD if they met the National Institute of Neurological and Communicative Disorders and Stroke/Alzheimer's Disease and Related Disorders Association (NINCDS-ADRDA) criteria [20]. The following patients were excluded from the study: (1) those whose dementia developed 12 months or later after the onset of Parkinson's disease; (2) those who fulfilled both probable AD and possible DLB criteria; (3) those with developmental abnormalities, serious psychiatric diseases, substance abuse, or significant neurologic antecedents, such as brain trauma, brain tumor, epilepsy, and inflammatory disease; (4) those who had associated delirium with dementia; (5) those in a nursing home, and (6) those without a reliable informant.

\section{Assessments and Measures}

The demographics of all patients were ascertained through clinical interviews of their caregivers, including previous diseases and drug history.

The severity of dementia was rated by using the Clinical Dementia Rating (CDR) [21], of which the scores range between 0 (no cognitive decline), 0.5 (questionable dementia), 1 (mild dementia), 2 (moderate dementia), and 3 (severe dementia). This study involved patients with a CDR score of 0.5 if they fulfilled the criteria of dementia; we considered these patients as having very mild dementia.

We assessed the patients' comprehensive BPSD semiquantitatively by an interview with their caregivers, using a Japanese version of the Neuropsychiatric Inventory (NPI) [22]. Using the NPI, the following 12 symptoms were rated on the basis of the patient's condition during the last month before the interview: delusions; hallucinations; agitation; depression (dysphoria); anxiety; euphoria; apathy; disinhibition; irritability and lability; aberrant motor behavior (AMB); sleep disturbances, and eating abnormalities. In this measurement, according to the criteria-based rating scheme, the severity of each manifestation was classified into 4 grades (from 1 to 3; 0 if absent), and the frequency of each manifestation was also classified into 5 grades (from 1 to 4; 0 if absent). The NPI score (severity $\times$ frequency) was calculated for each manifestation (range of possible scores, $0-12$ ). The maximum total score in the NPI for the 12 manifestations is 144 . The CDR and NPI assessments were performed within 2 months of the first visit.

\section{Statistical Analysis}

Demographic and clinical variables, the NPI total score, and the score of each individual NPI domain were evaluated between the DLB and AD groups with the $t$ test and the $\chi^{2}$ analysis. The relationship of BPSD with the type of dementia according to dementia severity was assessed in a two-way analysis of variance (ANOVA) model with the NPI total score as a dependent variable, type of dementia (AD versus DLB) and dementia severity (CDR 0.5 versus CDR 1 versus CDR 2) as main effects, and (type of dementia) $\times$ (dementia severity) as an interaction term. We did not include CDR 3 patients in the analysis because of the small number of patients. When significant effects were found, they were further examined with analysis of covariance (ANCOVA) in which age, sex, education, and presence or absence of cholinesterase inhibitor (ChEI) usage were included into the model as covariates. For all analyses, a p value $<0.05$ was regarded as statistically significant. For multiple comparisons, the $\alpha$ level was set at $0.004(0.05 / 12)$ in accordance with the Bonferroni adjustment. Statistical operations were performed with SPSS for Windows, version 17.0 (SPSS Inc., Chicago, Ill., USA). 
Dementia

and Geriatric

Cognitive Disorders

Table 1. Patient demographics and clinical characteristics

Table 2. Mean composite scores (frequency $\times$ severity) of individual NPI symptoms in patients with DLB and AD

\begin{tabular}{|c|c|c|c|}
\hline \multicolumn{4}{|c|}{ Dement Geriatr Cogn Disord Extra 2015;5:244-252 } \\
\hline \multicolumn{2}{|l|}{ DOI: 10.1159/000381800 } & \multicolumn{2}{|c|}{$\begin{array}{l}\text { (c) } 2015 \text { S. Karger AG, Basel } \\
\text { www.karger.com/dee }\end{array}$} \\
\hline \multicolumn{4}{|c|}{$\begin{array}{l}\text { Hashimoto et al.: Relationship between Dementia Severity and Behavioral and } \\
\text { Psychological Symptoms of Dementia in DLB and AD Patients }\end{array}$} \\
\hline & DLB $(n=97)$ & $A D(n=393)$ & $\mathrm{p}$ value \\
\hline Age, years & $77.6 \pm 5.4$ & $77.0 \pm 8.0$ & 0.52 \\
\hline Males/females & $45 / 52$ & $117 / 276$ & 0.002 \\
\hline Education, years & $10.3 \pm 2.7$ & $10.6 \pm 2.9$ & 0.31 \\
\hline MMSE score & $18.6 \pm 5.7$ & $19.0 \pm 5.4$ & 0.55 \\
\hline $\operatorname{CDR}(0.5 / 1 / 2 / 3)$ & $23 / 45 / 27 / 2$ & $112 / 181 / 87 / 13$ & 0.55 \\
\hline ChEI prescription & $46(47.4)$ & $138(35.1)$ & 0.03 \\
\hline
\end{tabular}

Values are $\mathrm{n}(\%)$ or the mean \pm SD.

\begin{tabular}{lccc}
\hline & DLB $(\mathrm{n}=97)$ & $\mathrm{AD}(\mathrm{n}=393)$ & p value $^{1}$ \\
\hline Delusions & $2.38 \pm 3.46$ & $1.17 \pm 2.30$ & $<0.001$ \\
Hallucinations & $3.28 \pm 3.82$ & $0.22 \pm 0.83$ & $<0.001$ \\
Agitation & $1.06 \pm 2.15$ & $1.27 \pm 2.25$ & 0.42 \\
Depression & $1.46 \pm 2.63$ & $0.92 \pm 1.80$ & 0.017 \\
Anxiety & $1.81 \pm 3.01$ & $1.02 \pm 2.01$ & 0.002 \\
Euphoria & $0.13 \pm 0.72$ & $0.07 \pm 0.48$ & 0.30 \\
Apathy & $4.56 \pm 4.03$ & $3.64 \pm 3.27$ & 0.019 \\
Disinhibition & $0.35 \pm 1.54$ & $0.37 \pm 1.33$ & 0.92 \\
Irritability & $1.19 \pm 2.56$ & $1.17 \pm 2.44$ & 0.96 \\
AMB & $1.54 \pm 3.21$ & $1.18 \pm 2.75$ & 0.27 \\
Sleep disturbances & $1.87 \pm 2.93$ & $0.92 \pm 1.98$ & $<0.001$ \\
Eating abnormalities & $1.84 \pm 3.50$ & $1.35 \pm 2.64$ & 0.14 \\
Total NPI score & $21.4 \pm 17.0$ & $13.3 \pm 12.1$ & $<0.001$ \\
\hline
\end{tabular}

Values are the mean \pm SD. ${ }^{1}$ t test

\section{Results}

Demographic variables of the DLB and AD groups are shown in table 1. No statistically significant differences were found in age, educational level, and disease severity represented by both the MMSE and CDR between the DLB and AD groups. The DLB group consisted of significantly more males and fewer females than the AD group. The proportion of patients receiving treatment with ChEIs was significantly higher in the DLB group (47.4\%) than in the AD group (35.1\%).

The NPI total score and the score of each individual NPI domain in the two groups are shown in table 2 . The NPI total score in the DLB group was significantly higher than that in the AD group $(\mathrm{p}<0.001)$. With regard to the NPI individual domain, the DLB group had significantly higher scores for delusions $(p<0.001)$, hallucinations $(p<0.001)$, anxiety $(p=0.002)$, and sleep disturbances $(\mathrm{p}<0.004)$ than the AD group.

Two-way ANOVA revealed that both the effect of the type of dementia and the effect of dementia severity were significant $(F=5.37, p<0.001$, and $F=4.39, p=0.013$, respectively). Moreover, the (type of dementia) $\times$ (dementia severity) interaction was significant $(\mathrm{F}=$ $5.37, p=0.005)$. As a significant (type of dementia) $\times$ (dementia severity) interaction was obtained, a further test with one-way ANOVA was conducted among three severity groups (i.e., $\operatorname{CDR}=0.5,1$, and 2) within each disease group for the NPI total score and each NPI subset score. Table 3 shows the demographic variables, the mean NPI total score, and the mean score of each NPI subset in the DLB and AD groups stratified by different severities. 
Table 3. Patient characteristics and mean scores of individual NPI domains according to dementia severity (CDR score) in patients with DLB and AD

\begin{tabular}{|c|c|c|c|c|c|c|c|c|c|}
\hline & \multicolumn{4}{|c|}{ CDR score in DLB } & \multicolumn{5}{|c|}{ CDR score in AD } \\
\hline & 0.5 & 1 & 2 & $\mathrm{p}$ value & 0.5 & 1 & 2 & $\mathrm{p}$ value & post hoc \\
\hline Patients (females) & 23 (13) & $45(23)$ & 27 (15) & & $112(74)$ & $181(134)$ & 87 (59) & & \\
\hline Age, years & $74.2 \pm 5.5$ & $77.8 \pm 5.3$ & $80.2 \pm 4.3$ & & $75.3 \pm 8.2$ & $76.9 \pm 7.9$ & $79.3 \pm 7.6$ & & \\
\hline Education, years & $10.5 \pm 2.8$ & $10.0 \pm 2.8$ & $10.5 \pm 2.4$ & & $11.4 \pm 2.7$ & $10.7 \pm 3.0$ & $9.4 \pm 2.5$ & & \\
\hline MMSE score & $23.0 \pm 3.7$ & $20.0 \pm 3.8$ & $13.6 \pm 4.9$ & & $22.7 \pm 2.8$ & $19.8 \pm 3.7$ & $14.3 \pm 4.7$ & & \\
\hline \multicolumn{10}{|l|}{ NPI domain } \\
\hline Delusions & $2.22 \pm 3.78$ & $2.62 \pm 3.55$ & $2.30 \pm 3.21$ & 0.88 & $0.64 \pm 1.94$ & $1.15 \pm 2.34$ & $1.87 \pm 2.50$ & 0.001 & $0.5<2$ \\
\hline Hallucinations & $2.96 \pm 3.89$ & $3.64 \pm 4.09$ & $3.07 \pm 3.49$ & 0.73 & $0.04 \pm 0.31$ & $0.18 \pm 0.82$ & $0.44 \pm 1.08$ & 0.002 & $0.5<2$ \\
\hline Agitation & $0.65 \pm 1.50$ & $0.84 \pm 2.02$ & $1.44 \pm 2.44$ & 0.34 & $0.50 \pm 1.36$ & $1.25 \pm 2.09$ & $2.08 \pm 2.70$ & $<0.001$ & $0.5<2$ \\
\hline Depression & $2.35 \pm 3.94$ & $1.31 \pm 2.30$ & $1.07 \pm 1.57$ & 0.20 & $0.71 \pm 1.56$ & $1.10 \pm 2.04$ & $0.86 \pm 1.59$ & 0.18 & \\
\hline Anxiety & $1.87 \pm 4.13$ & $1.84 \pm 2.95$ & $1.67 \pm 2.02$ & 0.96 & $0.60 \pm 1.54$ & $1.06 \pm 2.02$ & $1.16 \pm 1.98$ & 0.063 & \\
\hline Euphoria & $0.13 \pm 0.63$ & $0.02 \pm 0.15$ & $0.33 \pm 1.21$ & 0.21 & $0.01 \pm 0.09$ & $0.07 \pm 0.44$ & $0.03 \pm 0.18$ & 0.32 & \\
\hline Apathy & $4.09 \pm 4.80$ & $3.51 \pm 3.43$ & $6.30 \pm 3.59$ & 0.013 & $2.06 \pm 2.31$ & $3.92 \pm 3.17$ & $4.54 \pm 3.52$ & $<0.001$ & $0.5<1,2$ \\
\hline Disinhibition & $0.65 \pm 2.52$ & $0.09 \pm 0.60$ & $0.52 \pm 1.60$ & 0.30 & $0.11 \pm 0.69$ & $0.35 \pm 1.47$ & $0.59 \pm 1.36$ & 0.029 & \\
\hline Irritability & $1.26 \pm 2.47$ & $1.00 \pm 2.28$ & $1.52 \pm 3.17$ & 0.71 & $0.54 \pm 1.44$ & $1.15 \pm 2.39$ & $1.93 \pm 3.13$ & $<0.001$ & $0.5<2$ \\
\hline $\mathrm{AMB}$ & $1.39 \pm 3.69$ & $1.49 \pm 3.05$ & $1.74 \pm 3.25$ & 0.92 & $0.16 \pm 0.80$ & $1.22 \pm 2.87$ & $1.86 \pm 3.12$ & $<0.001$ & $0.5<1,2$ \\
\hline Sleep disturbance & $1.65 \pm 3.10$ & $1.77 \pm 2.80$ & $2.38 \pm 3.11$ & 0.65 & $0.48 \pm 1.30$ & $0.81 \pm 1.77$ & $1.43 \pm 2.42$ & 0.001 & $0.5<2$ \\
\hline Eating abnormalities & $2.26 \pm 4.48$ & $1.88 \pm 3.10$ & $1.59 \pm 3.35$ & 0.78 & $0.88 \pm 2.25$ & $1.42 \pm 2.73$ & $1.67 \pm 2.73$ & 0.08 & \\
\hline Total NPI score & $21.5 \pm 19.1$ & $19.9 \pm 17.1$ & $23.9 \pm 15.9$ & 0.64 & $6.70 \pm 7.5$ & $13.7 \pm 11.7$ & $18.4 \pm 11.7$ & $<0.001$ & $0.5<1<2$ \\
\hline
\end{tabular}

Fig. 1. Mean NPI total score according to dementia severity (CDR score) in the DLB and AD groups. ${ }^{*} \mathrm{p}<0.01$.

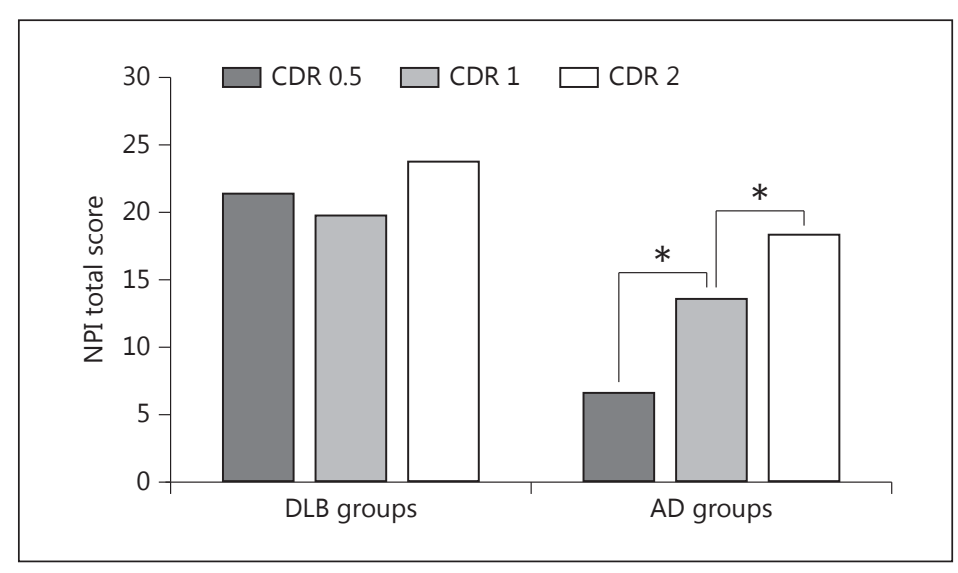

Significant CDR group differences were observed for the total NPI score and the scores of delusions, hallucinations, agitation, apathy, irritability, AMB, and sleep disturbance subdomains in AD (fig. 1). Tukey post hoc analysis showed that the scores for delusions, hallucinations, agitation, irritability, and sleep disturbances were significantly lower in the CDR 0.5 group than in the CDR 2 group. The scores of apathy and AMB were significantly lower in the CDR 0.5 group than in both the CDR 1 and CDR 2 groups. On the contrary, significant differences were not seen in the NPI total score or in any of the individual NPI domains across CDR staging. In a one-way ANCOVA, where age, sex, education, and ChEI administration were included in the model as covariates, the effects of CDR on NPI were unchanged in both the DLB and AD groups. 
Table 4. Comparison of mean scores of individual NPI domains between patients with DLB and AD within the same CDR score

\begin{tabular}{|c|c|c|c|c|c|c|c|c|c|}
\hline \multirow[t]{2}{*}{ NPI domain } & \multicolumn{3}{|c|}{ CDR score $=0.5$} & \multicolumn{3}{|c|}{ CDR score $=1$} & \multicolumn{3}{|c|}{ CDR score $=2$} \\
\hline & DLB & $\mathrm{AD}$ & $p$ value $^{1}$ & DLB & $\mathrm{AD}$ & p value ${ }^{1}$ & DLB & $\mathrm{AD}$ & p value ${ }^{1}$ \\
\hline Delusions & $2.22 \pm 3.78$ & $0.64 \pm 1.94$ & 0.004 & $2.62 \pm 3.55$ & $1.15 \pm 2.34$ & $<0.001$ & $2.30 \pm 3.21$ & $1.87 \pm 2.50$ & 0.48 \\
\hline Hallucinations & $2.96 \pm 3.89$ & $0.04 \pm 0.31$ & $<0.001$ & $3.64 \pm 4.09$ & $0.18 \pm 0.82$ & $<0.001$ & $3.07 \pm 3.49$ & $0.44 \pm 1.08$ & $<0.001$ \\
\hline Agitation & $0.65 \pm 1.50$ & $0.50 \pm 1.36$ & 0.63 & $0.84 \pm 2.02$ & $1.25 \pm 2.09$ & 0.24 & $1.44 \pm 2.44$ & $2.08 \pm 2.70$ & 0.28 \\
\hline Depression & $2.35 \pm 3.94$ & $0.71 \pm 1.56$ & 0.001 & $1.31 \pm 2.30$ & $1.10 \pm 2.04$ & 0.54 & $1.07 \pm 1.57$ & $0.86 \pm 1.59$ & 0.54 \\
\hline Anxiety & $1.87 \pm 4.13$ & $0.60 \pm 1.54$ & 0.012 & $1.84 \pm 2.95$ & $1.06 \pm 2.02$ & 0.035 & $1.67 \pm 2.02$ & $1.16 \pm 1.98$ & 0.25 \\
\hline Euphoria & $0.13 \pm 0.63$ & $0.01 \pm 0.09$ & 0.05 & $0.02 \pm 0.15$ & $0.07 \pm 0.44$ & 0.51 & $0.33 \pm 1.21$ & $0.03 \pm 0.18$ & 0.027 \\
\hline Apathy & $4.09 \pm 4.80$ & $2.06 \pm 2.31$ & 0.003 & $3.51 \pm 3.43$ & $3.92 \pm 3.17$ & 0.45 & $6.30 \pm 3.59$ & $4.54 \pm 3.52$ & 0.03 \\
\hline Disinhibition & $0.65 \pm 2.52$ & $0.11 \pm 0.69$ & 0.05 & $0.09 \pm 0.60$ & $0.35 \pm 1.47$ & 0.24 & $0.52 \pm 1.60$ & $0.59 \pm 1.36$ & 0.83 \\
\hline Irritability & $1.26 \pm 2.47$ & $0.54 \pm 1.44$ & 0.06 & $1.00 \pm 2.28$ & $1.15 \pm 2.39$ & 0.71 & $1.52 \pm 3.17$ & $1.93 \pm 3.13$ & 0.55 \\
\hline $\mathrm{AMB}$ & $1.39 \pm 3.69$ & $0.16 \pm 0.80$ & 0.002 & $1.49 \pm 3.05$ & $1.22 \pm 2.87$ & 0.57 & $1.74 \pm 3.25$ & $1.86 \pm 3.12$ & 0.86 \\
\hline Sleep disturbance & $1.65 \pm 3.10$ & $0.48 \pm 1.30$ & 0.004 & $1.77 \pm 2.80$ & $0.81 \pm 1.77$ & 0.005 & $2.38 \pm 3.11$ & $1.43 \pm 2.42$ & 0.10 \\
\hline Eating abnormalities & $2.26 \pm 4.48$ & $0.88 \pm 2.25$ & 0.03 & $1.88 \pm 3.10$ & $1.42 \pm 2.73$ & 0.41 & $1.59 \pm 3.35$ & $1.67 \pm 2.73$ & 0.90 \\
\hline Total NPI score & $21.5 \pm 19.1$ & $6.70 \pm 7.5$ & $<0.001$ & $19.9 \pm 17.1$ & $13.7 \pm 11.7$ & 0.004 & $23.9 \pm 15.9$ & $18.4 \pm 11.7$ & 0.057 \\
\hline
\end{tabular}

Values are the mean $\pm \mathrm{SD} .{ }^{1} \mathrm{t}$ test.

Furthermore, the mean NPI total score and the mean scores of each NPI subset were compared by the t test between patients with DLB and those with AD within each dementia stage. The results are shown in table 4. The NPI total score was significantly higher in the DLB group than in the AD group at the very mild dementia stage (CDR $=0.5$ ) and the mild dementia stage (CDR $=1$ ). Delusions, hallucinations, depression, apathy, AMB, and sleep disturbances were significantly more severe in patients with DLB than in those with AD at the very mild dementia stage $(C D R=0.5)$. At the mild dementia stage, delusions and hallucinations were more severe in patients with DLB than in those with AD. At the moderate dementia stage, no significant differences were found between the DLB and AD patients in the NPI total score or in any of the NPI subdomains except for hallucinations.

\section{Discussion}

The present study demonstrated that patients with DLB showed a higher severity of BPSD than patients with AD. Among the individual symptoms of BPSD, delusions, hallucinations, anxiety, and sleep disturbances were significantly more severe in the DLB group than in the $\mathrm{AD}$ group. These findings were consistent with previous reports $[15,23]$. The most remarkable finding of this study was that the severity of dementia, rated by CDR, was not always associated with worsening BPSD in DLB. The patients with DLB had profound BPSD even in the very mild dementia stage, and the degree of BPSD was unchanged until the moderate dementia stage. On the other hand, the severity of BPSD was related to dementia stage in the patients with $\mathrm{AD}$. The different relationships between dementia stage and severity of BPSD which emerged in the DLB and AD groups may reflect the different pathogenic mechanisms of BPSD in DLB and AD.

In DLB, the longitudinal course of BPSD has received little attention, and the results have been controversial. The present data support the results of a previous study reporting that depression, delusions, hallucinations, and delusional misidentification were equally common in different stages of DLB [13]. Unlike these studies, Caputo et al. [16] reported that the frequency of delusions, hallucinations, disinhibition, and sleep problems increased with the severity of dementia in DLB. The discrepancy between our findings and the findings of Caputo 
et al. [16] is probably due to the differences in methodological approaches. Caputo et al. [16] defined an NPI score of $\geq 4$ for any symptom as a clinically relevant disturbance and excluded subjects with milder BPSD from the analysis. In addition, differences in demographics could account for discrepancies, since our study subjects were mainly at the mild stage of dementia; the subjects in the Caputo study were mainly at the moderate stage. Indeed, in the Caputo report, the frequency of delusions and the frequency of disinhibition were significantly higher in subjects with severe dementia than in those with mild-to-moderate dementia; however, Caputo et al. [16] found no statistical differences between the subjects with mild dementia and those with moderate dementia.

The present data revealed that delusions, hallucinations, depression, apathy, AMB, and sleep disturbances were more severe in patients with DLB than in those with AD at the very mild dementia stage. A recent study comparing neuropsychiatric symptoms in patients with very mild DLB and AD by using the NPI reported that the DLB patients had a higher NPI total score and had significantly higher scores and frequencies for hallucinations, apathy, and sleep disturbances [24]. In addition, in a clinicopathological study, patients with mild cognitive impairment who subsequently developed DLB had more delirium and visual hallucinations compared with patients who later developed AD [25]. BPSD is reported to be the strongest determinant of the caregiver burden in patients with DLB [26]. Our data and these previous findings suggest that intensive management for BPSD should be provided from the very early stage to patients with DLB.

About $50 \%$ of patients with DLB have already been prescribed ChEIs at the initial assessment. A placebo-controlled, double-blind study for DLB showed that patients taking rivastigmine were significantly less apathetic and anxious, and had fewer delusions and hallucinations than controls [27]. In addition, a recent randomized, placebo-controlled trial for DLB reported that donepezil at doses of 5 and $10 \mathrm{mg} /$ day produced significant improvements in the NPI score, particularly in the delusion and hallucination domains [28]. The ChEI prescription rate was comparable among the three CDR groups in DLB, and even after controlling for this variable, the profile of BPSD remained unchanged. Therefore, the characteristic relationship between BPSD and dementia severity shown in patients with DLB cannot be attributed to intervention with ChEIs.

Although psychological and social factors contribute to the onset and persistence of BPSD in dementia [29], the different profiles of BPSD among the different dementia stages which emerge in DLB and AD may reflect differences in the underlying mechanisms of BPSD. Nagahama et al. [30] investigated the association between psychotic symptoms in DLB and brain perfusion on single photon emission computed tomography. These authors found that delusions of theft and persecution were associated with relative hyperperfusion in the frontal cortex. Furthermore, Miyazawa et al. [31] investigated the presence of hypermetabolism using positron emission tomography in patients with DLB and analyzed the relationships between the characteristics of DLB and hypermetabolism. These investigators showed a possible relationship between visual hallucination and hypermetabolism in the perimotor area, basal ganglia, and cerebellum. These neuroimaging findings suggest that the generation of delusions and visual hallucinations in patients with DLB may require functional preservation in the specific regions; this may be the reason for the present finding that the deterioration observed in dementia was not always associated with worsening psychotic symptoms in DLB.

The strength of the present study is that it was a large, prospective study using standardized assessment tools. However, several methodological issues limit the interpretation of the results of this study. First, the study samples were recruited from the first referrals to outpatient dementia clinics and excluded patients in a nursing home; this might have introduced a selection bias. In general, the deterioration of patients with dementia or with more severe BPSD increases caregiver stress and promotes nursing home placement. Our sample 
included only a small number of patients with severe dementia. Thus, our results cannot be generalized to all stages of dementia. Second, diagnosis was made solely on a clinical basis without histopathologic confirmation, with inevitably some uncertainty about the rate of misclassification. In addition, the presence of visual hallucinations or REM sleep behavior disorder is included in the criteria for probable DLB [19]. This may affect the apparent prevalence of these symptoms, and the effect should be discounted when interpreting the results. Third, the cross-sectional study design may not have been optimal since behavioral disturbances can fluctuate and may not be present at every examination [32]. Moreover, due to the cross-sectional study design, the relationship between the severity of dementia and BPSD expression could only be hypothesized. Longitudinal research may offer additional information on the development and course of BPSD in patients with dementia.

In conclusion, the results of this study suggested that the relationship of dementia stage with the expression of BPSD differed according to the type of dementia. BPSD and dementia severity were correlated in $\mathrm{AD}$ subjects, in whom psychiatric symptoms increase as the disease progresses; this was not found in DLB subjects. In DLB, severe BPSD emerged even at the very mild dementia stage, and the degree of BPSD was unchanged until the moderate dementia stage. These findings suggest the importance of taking into account both dementia type and dementia stage for managing BPSD.

\section{Acknowledgments}

The authors gratefully acknowledge the assistance of the staff of the Department of Neuropsychiatry, Faculty of Life Sciences, Kumamoto University, Kumamoto, Japan. The present study was undertaken with the support of grants provided by the Ministry of Health, Labour and Welfare (Research on Dementia; H21-Dementia-General-005) for Manabu Ikeda.

\section{Disclosure Statement}

The authors report no conflicts of interest.

\section{References}

1 Finkel SI, Costa e Silva J, Cohen G, Miller S, Sartorius N: Behavioral and psychological signs and symptoms of dementia: a consensus statement on current knowledge and implications for research and treatment. Int Psychogeriatr 1996;8(suppl 3):497-500.

-2 Carson S, McDonagh M, Peterson K: A systematic review of the efficacy and safety of atypical antipsychotics in patients with psychological and behavioral symptoms of dementia. J Am Geriatr Soc 2006;54:354-361.

-3 Stern Y, Tang MX, Albert MS, Brandt J, Jacobs DM, Bell K, Marder K, Sano M, Devanand D, Albert SM, Bylsma F, Tsai WY: Predicting time to nursing home care and death in individuals with Alzheimer disease. JAMA 1997; 277:806-812.

4 Lyketsos CG, Steele C, Baker L, Galik E, Kopunek S, Steinberg M, Warren A: Major and minor depression in Alzheimer's disease: prevalence and impact. J Neuropsychiatry Clin Neurosci 1997;9:556-561.

5 González-Salvador T, Lyketsos CG, Baker A, Hovanec L, Roques C, Brandt J, Steele C: Quality of life in dementia patients in long-term care. Int J Geriatr Psychiatry 2000;15:181-189.

6 Steele C, Rovner B, Chase GA, Folstein M: Psychiatric symptoms and nursing home placement of patients with Alzheimer's disease. Am J Psychiatry 1990;147:1049-1051.

7 Harwood DG, Barker WW, Ownby R, Duara R: Relationship of behavioral and psychological symptoms to cognitive impairment and functional status in Alzheimer's disease. Int J Geriatr Psychiatry 2000;15:393-400.

-8 Lopez OL, Becker JT, Sweet RA, Klunk W, Kaufer DI, Saxton J, Habeych M, DeKosky ST: Psychiatric symptoms vary with the severity of dementia in probable Alzheimer's disease. J Neuropsychiatry Clin Neurosci 2003;15: 346-353. 


\section{DOI: $10.1159 / 000381800$}

Hashimoto et al.: Relationship between Dementia Severity and Behavioral and

Psychological Symptoms of Dementia in DLB and AD Patients

-9 Srikanth S, Nagaraja AV, Ratnavalli E: Neuropsychiatric symptoms in dementia-frequency, relationship to dementia severity and comparison in Alzheimer's disease, vascular dementia and frontotemporal dementia. J Neurol Sci 2005;236:43-48.

10 Thompson C, Brodaty H, Trollor J, Sachdev P: Behavioral and psychological symptoms associated with dementia subtype and severity. Int Psychogeriatr 2010;22:300-305.

-11 McKeith IG, Burn DJ, Ballard CG, Collerton D, Jaros E, Morris CM, McLaren A, Perry EK, Perry R, Piggott MA, O’Brien JT: Dementia with Lewy bodies. Semin Clin Neuropsychiatry 2003;8:46-57.

12 Buracchio T, Arvanitakis Z, Gorbien M: Dementia with Lewy bodies: current concepts. Dement Geriatr Cogn Disord 2005;20:306-320.

13 Ballard C, Holmes C, McKeith I, Neill D, O’Brien J, Cairns N, Lantos P, Perry E, Ince P, Perry R: Psychiatric morbidity in dementia with Lewy bodies: a prospective clinical and neuropathological comparative study with Alzheimer's disease. Am J Psychiatry 1999;156:1039-1045.

14 Hirono N, Cummings JL: Neuropsychiatric aspects of dementia with Lewy bodies. Curr Psychiatry Rep 1999; 1:85-92.

15 Del Ser T, McKeith I, Anand R, Cicin-Sain A, Ferrara R, Spiegel R: Dementia with Lewy bodies: finding from an international multicentre study. Int J Geriatr Psychiatry 2000;15:1034-1045.

16 Caputo M, Monastero R, Mariani E, Santucci A, Mangialasche F, Camarda R, Senin U, Mecocci P: Neuropsychiatric symptoms in 921 elderly subjects with dementia: a comparison between vascular and neurodegenerative types. Acta Psychiatr Scand 2008;117:455-464.

-17 Folstein MF, Folstein SE, McHugh PR: 'Mini-mental state'. A practical method for grading the cognitive state of patients for the clinician. J Psychiatr Res 1975;12:189-198.

18 American Psychiatric Association: Diagnostic and Statistical Manual on Mental Disorders, ed 3, revised (DSMIII-R). Washington, American Psychiatric Association, 1987.

19 McKeith IG, Dickson DW, Lowe J, Emre M, O’Brien JT, Feldman H, Cummings J, Duda JE, Lippa C, Perry EK, Aarsland D, Arai H, Ballard CG, Boeve B, Burn DJ, Costa D, Del Ser T, Dubois B, Galasko D, Gauthier S, Goetz CG, Gomez-Tortosa E, Halliday G, Hansen LA, Hardy J, Iwatsubo T, Kalaria RN, Kaufer D, Kenny RA, Korczyn A, Kosaka K, Lee VM, Lees A, Litvan I, Londos E, Lopez OL, Minoshima S, Mizuno Y, Molina JA, Mukaetova-Ladinska EB, Pasquier F, Perry RH, Schulz JB, Trojanowski JQ, Yamada M; Consortium on DLB: Diagnosis and management of dementia with Lewy bodies: third report of the DLB Consortium. Neurology 2005;65:1863-1872.

20 McKhann G, Drachman D, Folstein M, Katzman R, Price D, Stadlan EM: Clinical diagnosis of Alzheimer's disease: report of the NINCDS-ADRDA Work Group under the auspices of Department of Health and Human Services Task Force on Alzheimer's Disease. Neurology 1984;34:939-944.

21 Hughes CP, Berg L, Danziger WL, Coben LA, Martin RL: A new clinical scale for the staging of dementia. Br J Psychiatry 1982;140:566-572.

-22 Cummings JL: The Neuropsychiatric Inventory: assessing psychopathology in dementia patients. Neurology 1997;48(suppl 6):10-16.

-23 McKeith I, Mintzer J, Aarsland D, Burn D, Chiu H, Cohen-Mansfield J, Dickson D, Dubois B, Duda JE, Feldman H, Gauthier S, Halliday G, Lawlor B, Lippa C, Lopez OL, Carlos Machado J, O’Brien J, Playfer J, Reid W; International Psychogeriatric Association Expert Meeting on DLB: Dementia with Lewy bodies. Lancet Neurol 2004;3: 19-28.

-24 Bjoerke-Bertheussen J, Ehrt U, Rongve A, Ballard C, Aarsland D: Neuropsychiatric symptoms in mild dementia with Lewy bodies and Alzheimer's disease. Dement Geriatr Cogn Disord 2012;34:1-6.

25 Molano J, Boeve B, Ferman T, Smith G, Parisi J, Dickson D, Knopman D, Graff-Radford N, Geda Y, Lucas J, Kantarci K, Shiung M, Jack C, Silber M, Pankratz VS, Petersen R: Mild cognitive impairment associated with limbic and neocortical Lewy body disease: a clinicopathological study. Brain 2010;133:540-556.

26 Leggett AN, Zarit S, Taylor A, Galvin JE: Stress and burden among caregivers of patients with Lewy body dementia. Gerontologist 2011;51:76-85.

27 McKeith I, Del Ser T, Spano P, Emre M, Wesnes K, Anand R, Cicin-Sain A, Ferrara R, Spiegel R: Efficacy of rivastigmine in dementia with Lewy bodies: a randomised, double-blind, placebo-controlled international study. Lancet 2000;356:2031-2036.

28 Mori E, Ikeda M, Kosaka K; Donepezil-DLB Study Investigators: Donepezil for dementia with Lewy bodies: a randomized, placebo-controlled trial. Ann Neurol 2012;72:41-52.

29 Aarsland D, Sharp S, Ballard C: Psychiatric and behavioral symptoms in Alzheimer's disease and other dementias: etiology and management. Curr Neurol Neurosci Rep 2005;5:345-354.

-30 Nagahama Y, Okina T, Suzuki N, Matsuda M: Neural correlates of psychotic symptoms in dementia with Lewy bodies. Brain 2010;133:557-567.

-31 Miyazawa N, Shinohara T, Nagasaka T, Hayashi M: Hypermetabolism in patients with dementia with Lewy bodies. Clin Nucl Med 2010;35:490-493.

32 Aalten P, de Vugt ME, Jaspers N, Jolles J, Verhey FR: The course of neuropsychiatric symptoms in dementia. Part I: findings from the two-year longitudinal Maasbed study. Int J Geriatr Psychiatry 2005;20:523-530. 